\title{
TSH mediates increased risk of PTC in Hashimoto thyroiditis
}

The frequency of papillary thyroid carcinoma (PTC) is increased in patients with nodular Hashimoto thyroiditis, report researchers from the University of Pisa. They also show that increased TSH levels contribute to increased risk of PTC.

The relationship between Hashimoto thyroiditis and PTC is controversial. The researchers used cytology to assess the presence of PTC in 13,738 patients with thyroid nodules, some of whom were receiving treatment with levothyroxine. Patients were classified as having nodular Hashimoto thyroiditis or nodular goiter according to the presence of hypothyroidism, level of serum thyroid autoantibodies and/or ultrasonography findings. Patients with nodular goiter were divided into two groups according to the presence $\left(\mathrm{TAb}^{+}\right)$or undetectability $\left(\mathrm{TAb}^{-}\right)$ of thyroid autoantibodies.
Among the 9,824 patients not receiving levothyroxine, 9.1\% had nodular Hashimoto thyroiditis, $24.0 \%$ had $\mathrm{TAb}^{+}$ nodular goiter and $66.9 \%$ had $\mathrm{TAb}^{-}$nodular goiter. In patients with nodular Hashimoto thyroiditis, the frequency of PTC was significantly higher than in patients with nodular goiter. In addition, patients with nodular Hashimoto thyroiditis also had higher levels of TSH in serum. Increased TSH levels were a strong independent predictor of the probability of having PTC (OR 1.111 per $1 \mu \mathrm{U} / \mathrm{ml}$ ). Thyroglobulin antibody levels slightly increased the risk of PTC, whereas thyroperoxidase antibody levels were not related to PTC.

Similarly, among the 3,914 patients taking levothyroxine, the frequency of PTC was significantly higher among those with nodular Hashimoto thyroiditis (16.3\%) than among those with nodular goiter, be it $\mathrm{TAb}^{+}(26.4 \%)$ or $\mathrm{TAb}^{-}(57.3 \%)$.
The difference between the groups was not significant, however, among patients with serum TSH levels below $0.90 \mu \mathrm{U} / \mathrm{ml}$.

As the authors acknowledge, the duration of levothyroxine therapy and persistence of low TSH levels were not assessed in this cross-sectional study. Longitudinal case-control studies would be required to address these issues.

The results of the study suggest that "in patients with nodular Hashimoto thyroiditis, treatment with levothyroxine-which reduces TSH levels-may reduce the frequency of PTC, as already reported in patients with nodular goiter," conclude the researchers.

Joana Osório

Original article Fiore, E. et al. Hashimoto's thyroiditis is associated with papillary thyroid carcinoma: role of TSH and of treatment with L-thyroxin. Endocr. Relat. Cancer doi:10.1530/ERC-11-0028 